\title{
МЕТОДИЧЕСКОЕ И АЛГОРИТМИЧЕСКОЕ ОБЕСПЕЧЕНИЕ АВТОМАТИЗИРОВАННОЙ ЭКСПЕРИМЕНТАЛЬНОЙ УСТАНОВКИ УДАЛЕНИЯ ГИДРАТОВ В ГАЗОКОНДЕНСАТНОЙ СМЕСИ В ТРУБОПРОВОДЕ
}

\author{
Волков Павел Валерьевич1,2, \\ VolkovPV@tomsknipi.ru
}

\author{
Большунов Артем Владимирович², \\ tpuiprgrnm@mail.ru
}

\author{
Мостокалов Кирилл Алексеевич², \\ $555 @ t-s k . r u$ \\ 1 АО ТомскНИПИнефть, \\ Россия, 634027, г. Томск, пр. Мира, 72. \\ 2 Национальный исследовательский Томский политехнический университет, \\ Россия, 634050, г. Томск, пр. Ленина, 30.
}

\begin{abstract}
Актуальность исследования обусловлена тем, что в настоящее время в мировой нефтегазовой промышленности проблема образования гидратов стоит достаточно остро. Аккумулирование гидратов на участке трубопровода или в определенных узлах установок подготовки углеводородов может привести к срыву технологического процесса, выходу из строя оборудования, снижению качества готовой продукции для потребителя. Главная задача состоит в том, чтобы своевременно определять наличие гидратов в полости трубопровода или правильно прогнозировать процесс его формирования и аккумулирования. Существует множество методов борьбы с гидратообразованием, но они могут быть высокозатратны, малоэффективны или иметь высокий класс опасности. В статье рассмотрен новый способ борьбы с гидратообразованием и предложена методика по автоматизации процесса. Аппаратная часть рассматривается как имитационньй экспериментальньй стенд для проведения экспериментов, проверки аналитических расчетов и автоматизаиии. Программная часть представляет методику автоматизации определения зон возможного гидратообразования, что позволяет проводить более качественную оценку, и программу для автоматизированной работы опытно-промышленного образца в реальных условиях. Описаны алгоритмы работы, алгоритмы аварийной и нормальной остановки. Для более точного и быстрого анализа проблемных зон гидратообразования, а также безаварийной работы установки разработан строгий алгоритм. В систему команд алгоритма заложены все аварийные ситуации, возможные ошибки, предельные режимы работы. Работа всех алгоритмов была проверена практическим путем посредством имитации процесса реальной работы системы и подачи условных сигналов на вход в программный модуль установки.
\end{abstract}

Цель: применение и анализ работы алгоритмического обеспечения для работы экспериментальной установки по удалению гидратов из потока газоконденсатной смеси.

Объект: автономная модульная установка для борьбы с гидратообазованием.

Методы: теоретические методы для обоснования закономерности течения процессов тепломассообмена, расчетноаналитический метод для определения качества работы разработанного программного обеспечения, практические методы по проведению экспериментов.

Результаты. Построен лабораторный стенд на базе Национального исследовательского Томского политехнического университета, проведены эксперименты для различных потоков газа с разными термобарическими условиями, разработано математическое и алгоритмическое обеспечение для автоматизации процесса работы установки, проведена проверка работы системы.

\section{Ключевые слова:}

Гидратообразование, алгоритм, программный комплекс, аккумулирование гидратов, циклон.

\section{Введение}

Открытие соединений гидратов произошло в XIX в. [1]. Тогда главной задачей было определить способ их образования, гидратообразующие вещества условия их диссоциации $[2,3]$. На сегодняшний день кристаллы гидратов (соединения молекул газа и капельной влаги) очень хорошо изучены, определены вещества, которые могут их образовывать. По своим физическим свойствам гидраты очень близки к кристаллам льда. В промышленности особый интерес имеется к гидратам газов, в частности ряда $\mathrm{C} 1$ и $\mathrm{C} 2$, которые встречаются наиболее часто $[4,5]$. Для образования гидратов достаточно трёх условий: 1) необходимое количество воды в системе, при высоком со- держании воды во флюиде гидраты образовываться не будут; 2) присутствие в компонентном составе гидратообразующего вещества; 3) благоприятные термобарические условия, а именно низкая температура и высокое давление [6, 7] (рис. 1).

Сам процесс образования гидратов для промышленности не так страшен, как их аккумулирование. Гидраты могут аккумулироваться на проблемных участках трубопроводов, например, в низменностях, относительно пути прокладки трубопровода или поворотах и отводах, что может привести к закупориванию трубопровода [8]. В этом случае применяются различные технологические методы для предотвращения гидратообразования, например, снижение дав- 
ления или закачка метанола в трубопровод [9]. Также гидраты могут оседать в клапанных сборках, забивать отборочные трубки приборов и приводить к их неисправности. Данный факт может очень пагубно сказаться на технологическом процессе, соответственно, снизить качество подготавливаемого продукта $[10,11]$. Поэтому актуальной задачей является именно технология прогнозирования образования гидратов в системе с целью недопущения их дальнейшего аккумулирования.

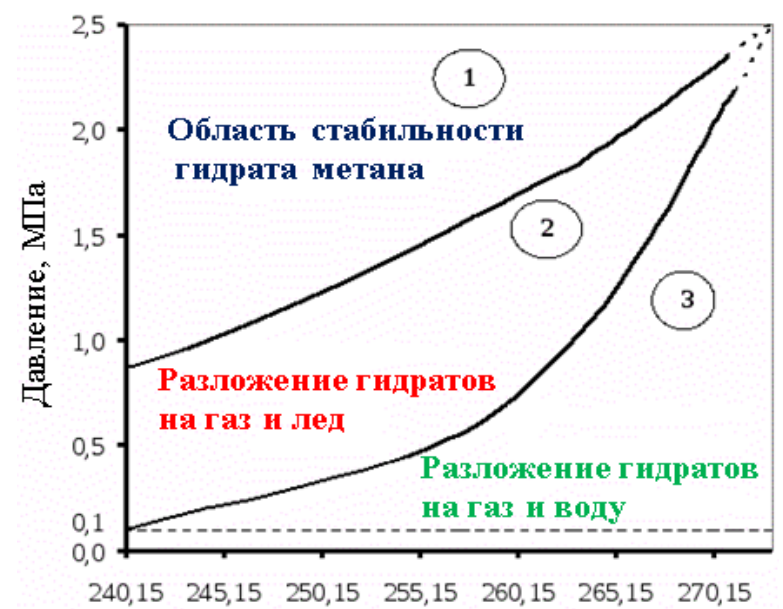

Температура, $\mathrm{K}$

Pис. 1. Диаграмма образования гидратов метана в зависимости от термобарических условий [7]

Fig. 1. Diagram of formation of methane hydrates depending on temperature and pressure conditions [7]

\section{Технология оценки прогноза образования гидратов}

Анализ возможности образования гидратов производился на основе уравнений по модели критерия Шухова. Сама аналитическая модель относительно компонентного состава, начальных условий и физических параметров системы сравнивает температуры системы, а именно: температуру газа (1), температуру точки росы (2) и температуру гидратообразования (2) относительно входного компонентного состава [12].

$$
T_{X}=T_{0}+\left(T_{\mathrm{H}}-T_{0}\right) e^{-\varpi_{\mathrm{y}} \frac{x}{l_{\mathrm{KC}}}}-D_{i} \frac{P_{\mathrm{H}}^{2}-P_{\mathrm{K}}^{2}}{2 a_{t} l_{\mathrm{KC}} P_{\mathrm{CP}}}\left(1-e^{-\varpi_{\mathrm{y}} \frac{x}{l_{\mathrm{KC}}}}\right),(1)
$$

где $T_{0}$ - температура окружающей среды; $T_{\mathrm{H}}$

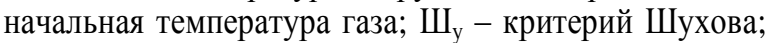
$D_{i}$ - коэффициент Джоуля-Томсона; $P_{\mathrm{H}}-$ начальное давление в трубопроводе; $P_{\mathrm{K}}-$ конечное давление в трубопроводе; $a_{t}-$ коэффициент Шухова; $x / l_{\mathrm{KC}}-$ уча-

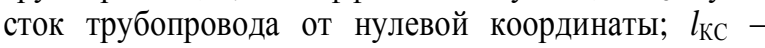
длинна трубопровода.

$$
T_{\mathrm{P}}=282,8 p_{x}^{0,0503} W^{0,0564},
$$

где $p_{x}$ - давление в точке трубопровода; $W$ - значение влагосодержания насыщенного газа.

$$
T_{\text {РГ }}=291,8-F_{0}+8,03 \ln p(x),
$$

где $F_{0}$ - функция приведенной плотности газа.

Основные коэффициенты, такие как критерий Шухова (4), значение влагосодержания насыщенного газа $(W)$, функция приведенной плотности газа (5), приведенная относительная плотность гидратообразующих компонентов газа (6), рассчитываются по формулам ниже:

$$
\amalg_{\mathrm{y}}=a_{t} l_{\mathrm{KC}}=225,5 \cdot 10^{6} \cdot \frac{K_{\mathrm{CP}} D_{\mathrm{вH}}}{Q \Delta C_{\mathrm{P}}} l_{\mathrm{KC}},
$$

где $K_{\mathrm{CP}}-$ средний коэффициент теплопередачи; $D_{\text {вн }}-$ внутренний диаметр трубопровода; $Q$ - расход газа при стандартных условиях; $C_{\mathrm{P}}-$ теплоемкость газа; $\Delta$ - относительная плотность.

$$
W=\left(\frac{T_{\mathrm{P}}}{282,8 p^{0,0503}}\right)^{\frac{1}{0,0564}},
$$

где $T_{\mathrm{P}}$ - температура, при которой начальное влагосодержание соответствует точке росы; $p$ - начальное давление в трубопроводе.

$$
F_{0}=9,21(\bar{\rho}-0,546)^{-0,225},
$$

где $\bar{\rho}$ - приведенная относительная плотность гидратообразующих компонентов газа.

$$
\bar{\rho}=\frac{\int_{i=1}^{k} a_{i} \Delta_{i}}{\int_{i=1}^{k} a_{i}},
$$

где $k$ - число гидратообразующих компонентов в газовой смеси; $a_{i}$ - объемная доля $i$-го гидратообразующего компонента в исходном газе; $\Delta_{i}$ - относительная плотность $i$-го гидратообразующего компонента.

При использовании расчета по критерию Шухова можно проводить адаптивные прогнозы, так как со временем компонентный состав флюида меняется, следовательно, меняются и зоны начала образования гидратов $[13,14]$. Зная геологическую модель месторождения, изучив стратегию разработки, можно иметь представление об изменении компонентного состава на ближайшие годы и провести расчет анализа зон по годам.

\section{Аналитический расчет}

Были рассмотрены начальные условия и реальный компонентный состав действующего трубопровода: $\omega\left(\mathrm{CH}_{4}\right)=82,94 \% ; \omega\left(\mathrm{C}_{2} \mathrm{H}_{6}\right)=5,82 \% ; \omega\left(\mathrm{C}_{3} \mathrm{H}_{8}\right)=5,03 \%$; $\omega\left(\mathrm{C}_{4} \mathrm{H}_{10}\right)=1,94 \% ; \omega\left(\mathrm{C}_{5} \mathrm{H}_{12}\right)=0,57 \% ; \omega\left(\mathrm{H}_{2} \mathrm{O}\right)=1,47 \%$; $\omega\left(\mathrm{CO}_{2}\right)=1,58 \% ; \omega\left(\mathrm{N}_{2}\right)=0,65 \%$. Параметры: плотность газа $0,858 \mathrm{\kappa} / \mathrm{m}^{3}$; относительная плотность газа по

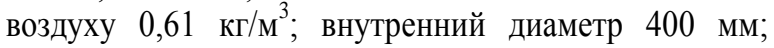
длинна трубопровода 142 км; расход газа 6823 тыс. $\mathrm{M}^{3} /$ сут, начальное давление 6,6 МПа, конечное давление 3,1 МПа; начальная температура газа 315 К; температура окружающей среды 276 К.

При проведении аналитического расчета по формулам на основе модели Критерия Шухова был получен графический результат (рис. 2). По его результатам была проведена интерпретация полученных данных.

По данным графика видно, что на данном этапе эксплуатации начало зоны образования гидратов находится примерно на 70-ом км трубопровода. Вы- 
вод основан на том, что температура газа на этом участке стала ниже температуры гидратообразования для данного компонентного состава и начальных термобарических и физических параметров. Снижение температуры газа произошло вследствие теплообмена с окружающей средой в отсутствии дополнительных источников тепла извне. Необходимо рассматривать применение технологических мер для недопущения аккумулирования гидратообразования [15].

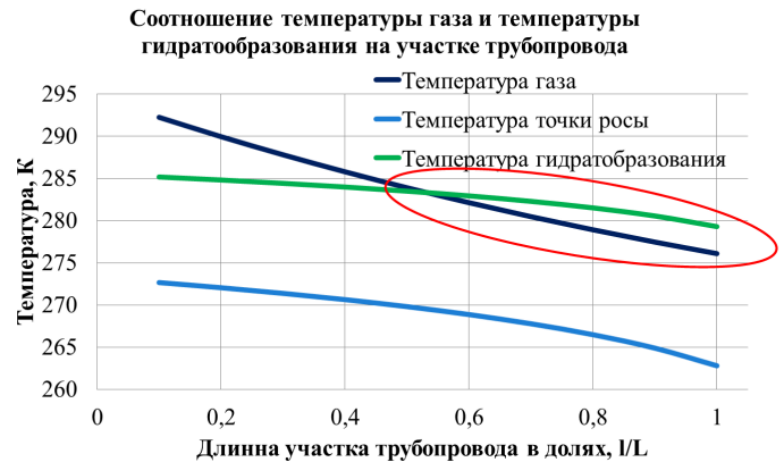

Pис. 2. Сводный график температур

Fig. 2. Temperature summary graph

\section{Метод борьбы с гидратообразованием}

На площадке Томского политехнического университета был разработан тепловой газодинамический метод борьбы с гидратообразованием и создан экспериментальный стенд, принципиальная схема представлена на рис. 3.

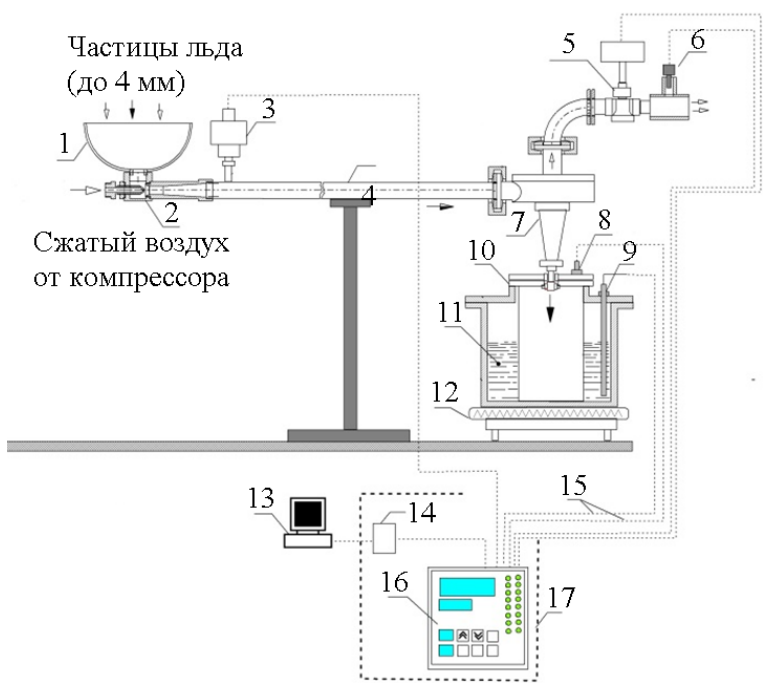

Рис. 3. Принципиальная схема лабораторной установки Fig. 3. Schematic diagram of a laboratory plant

Схема экспериментальной установки: 1 - приемная воронка; 2 - эжектор с кольцевым соплом; 3 электронный датчик давления, (4-20 мА); 4 - труба транспортная Dy 20 мм; 5 - электронный датчик разности давлений (ДМ5007А-ДД); 6 - совмещенный датчик влажности и температуры, ДВТ-02, (4-20 мА); 7 - вихревой циклон; 8 - датчик температуры газа (ДТС014-50М.В3.20/1); 9 - датчик температуры жидкости (ДТС014-50М.В3.20/1); 10 - тепловой форбун- кер; 11 - жидкость (глицерин); 12 - нагреватель 220 В; 13 - компьютер; 14 - преобразователь интерфейса RS-485 (AC-4); 15 - электрические линии связи; 16 модуль ввода аналоговый МВ 110-224.8А; 17 - шкаф системы управления.

Экспериментальный стенд создан для проведения лабораторных исследований и выдачи рекомендаций для создания опытно-промышленного образца.

\section{Принцип работы метода теплового} газодинамического воздействия

На вход в установку подается воздух под давлением до 0,5 МПа, параллельно с потоком подается лед (вещество, которое максимально приближено к свойствам гидрата в реальной системе). Температура окружающей среды во время проведения эксперимента составляет плюс $25^{\circ} \mathrm{C}$, поэтому часть льда превращается в воду. В данном случае имитируется реальная среда - газоконденсатный поток с частицами гидратов. Далее трехфазная система поступает на прямоточный циклон, где твердые частицы отделяются и попадают в тепловую камеру, а поток газожидкостной смеси следует дальше. В это время частицы льда (гидрата), попадая в тепловую камеру, плавятся и превращаются в воду, затем поступают посредством эжекции в основной поток и следуют дальше. Задача метода: превратить трехфазную систему в двухфазную и не допустить скопления льда в системе по ходу движения потока смеси в трубопроводе.

Источником энергии для тепловой камеры служит электрический ТЭН (трубчатый электронагреватель). Электроэнергия на установку подается централизовано. В промышленности установка модуля рассматривается на байпасной линии для возможности чистки трубопровода по основной линии и проведения других технологических операций $[16,17]$. В случае аварийного отключения предусмотрен определенный алгоритм остановки модуля: сначала открывается основная линия, затем закрывается байпасная линия (на которой установлено оборудование) до вывода из ремонта или устранения неисправностей.

Расчет зон начала гидратообразования необходимо проводить большое количество раз с изменением входных данных, следовательно, программа должна быть портативной, с понятным интерфейсом, не требующая высоких системных требований устройства.

Для решения этой задачи был разработан «Программно-аналитический комплекс для анализа зон возможного гидратообразования на участке трубопровода» [18]. Программа определяет числовое значение температуры газа, температуру точки росы газа и температуру начала гидратообразования для данной смеси. После интерпретации графических данных инженер может определить зоны образования гидратов на участке. Для решения задачи необходимо ввести исходные данные компонентного состава, а также начальные термобарические параметры смеси и геометрические характеристики трубопровода.

Компонентный состав смеси может резко меняться из-за смены температуры окружающей среды, изме- 
нения технологии добычи углеводородов, снижения расхода на одном и том же трубопроводе [19]. В таком случае при помощи программы можно выполнить быстрый перерасчет и получить новый графический результат для дальнейшей интерпретации.

Для недопущения аккумулирования гидратов, зная участок начала их образования, необходимо применить технологический метод [20]. Главная задача этого метода - сохранение двухфазной системы (газ и жидкость).
В настоящее время было проведено более пятисот экспериментов и доказана степень отделения льда из системы 85-93 \%. Для проведения более точных исследований и возможности снятия показаний параметров в реальном времени стенд был автоматизирован: установлены датчики температуры, давления, влажности, расходомер.

Все данные передаются через сетевой шлюз ПВ210 на облачный сервис OwenCloud, где отображаются все значения параметров, отображаются графики ведения процесса (рис. 4).

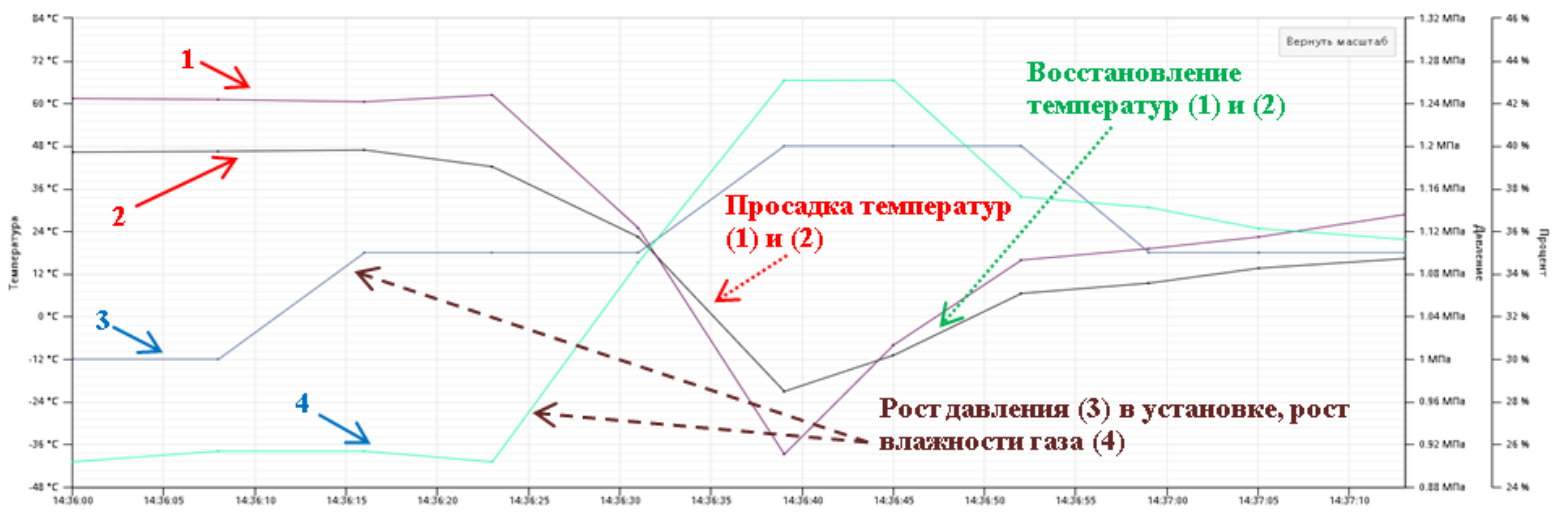

Puc. 4. Интерпретаиия данных с OwenCloud

Fig. 4. Interpreting data with OwenCloud

Обозначения на графике: 1 - температура теплоносителя; 2 - температура в тепловой камере; 3 - давление; 4 - влажность. Во время проведения эксперимента на установку подается давление от компрессо$\mathrm{pa}$, что эмитирует запуск трубопровода в работу, что способствует росту значения давления -3 . На постоянном уровне поддерживаются температуры теплоносителя и, соответственно, тепловой камеры $-1,2$. На вход в экспериментальную установку подается лед, часть которого начинается таять, что приводит к росту влажности - 4. Далее трехфазная смесь попадает на вихревой циклон, лед отделяется, попадает в тепловую камеру и под действием температуры плавится, что приводит к резкому скачку температур вниз. После того, как лед расплавился, температура начинает рост до прежнего значения. Задача программного комплекса: поддерживать мощность нагревателя, чтобы время восстановления температуры стремилось к 0 .

Bce датчики, блоки питания, модуль аналогово ввода и Wi-fi шлюз собраны в блоке управления, схема представлена на рис. 5.

Данные с датчиков передают сигнал на модуль аналогово ввода MB 110-8А, где сигнал обрабатывается и передается на преобразователь интерфейса RS-4 [21]. В первом варианте данные от RS-4 передаются непосредственно на персональный компьютер для анализа и интерпретации данных. Во втором варианте данные с RS-4 (интерфейс Recommended Standard 485) передаются на сетевой шлюз ПВ-210 и посредством интернет связи выгружаются в личный кабинет облачного сервиса OwenCloud. В данном сервисе отображаются все настройки, режимы работы приборов, данные с них, строятся графики в режиме реального времени для интерпретации.

Обозначения на рис. 5: ПК - персональный компьютер; RS 4 - преобразователь интерфейса RS-485; ПВ-210 - сетевой шлюз; Д1, Д2 - датчики температуры газа (ДТС014-50М.В3.20/1); Д3 - электронный датчик давления; Д4 - совмещенный датчик влажности и температуры (СДВТ-02).

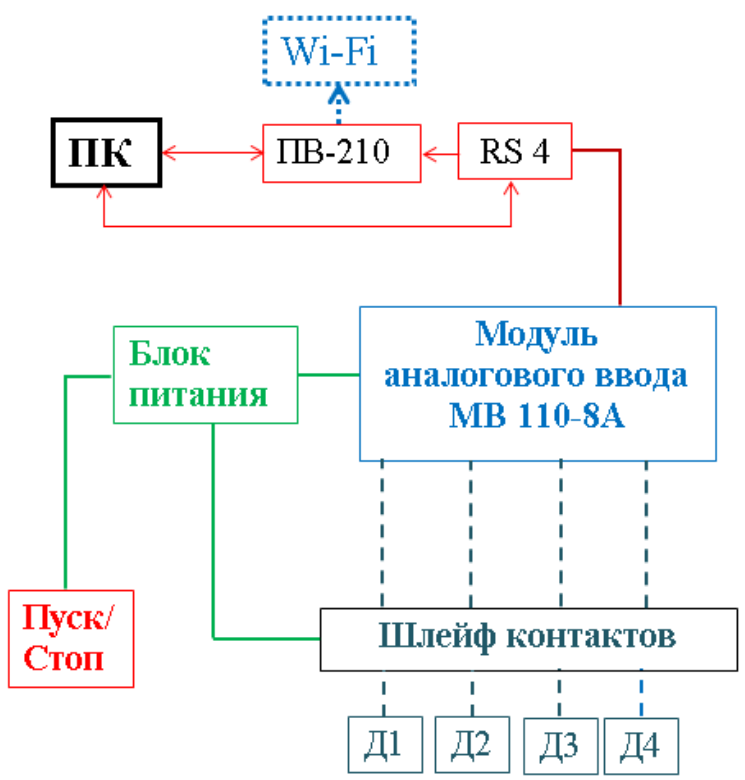

Рис. 5. Схема взаимосвязей приборов и блоков в шкафу управления

Fig. 5. Diagram of the interconnection of devices and blocks in the control panel 
Автоматизация работы экспериментальной установки для борьбы с гидратообразованием

Для автоматизированной работы установки разработан «Программный комплекс для автоматизации работы модульной вставки борьбы с гидратообразованием тепловым газодинамическим методом» [22]. Программа полностью управляет всеми параметрами установки, основным из которых является температура теплоносителя в тепловой камере. Данный параметр регулируется относительно времени восстановления заданной температуры и изменяет мощность нагревателя с целью стремления её к нулю [23]. Для работы модульной установки в промышленности программа предлагает ввести значения контролируемых параметров (температура, давление, влажность, расход). Также при ведении технологического процесса программное обеспечение выдает предупредительные сигналы о превышении значений верхний или нижних границ и отрабатывает алгоритм остановки при превышении пороговых значений блокирующих позиций (рис. 6). При достижении пороговых значений для позиций, которые не являются блокирующими, проходит предупредительная сигнализация, без остановки работы установки.

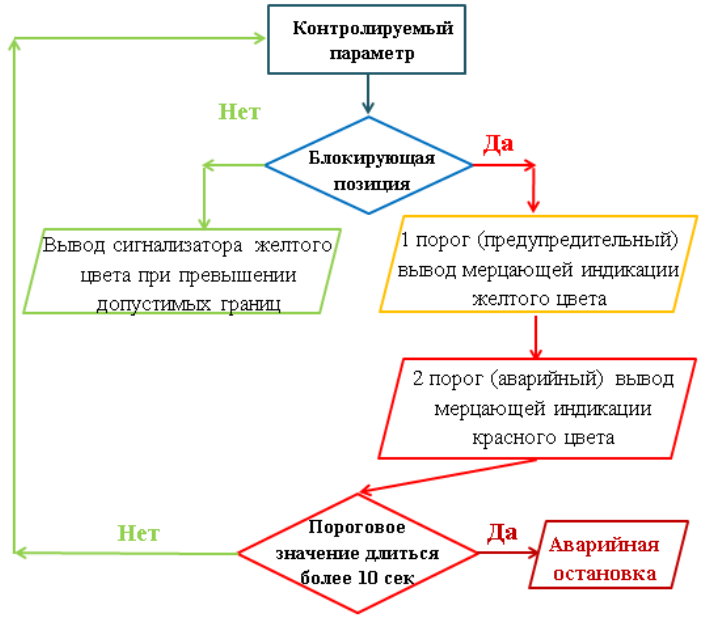

Pис. 6. Алгоритм аварийной ситуации

Fig. 6. Emergency algorithm

На (рис. 7) приведен пример работы программы, значения на вход подавались при помощи симулятора.

Экспериментальный стенд разработан при поддержке грантовой программы нефтегазовой компании British Petroleum.

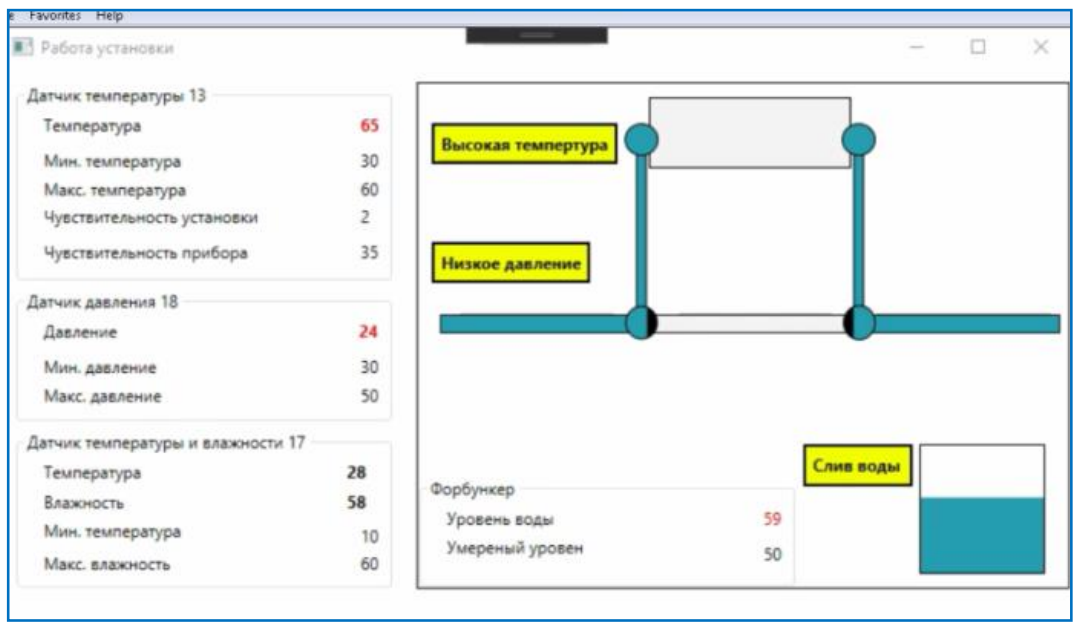

Рис. 7. Нормальный режим работы установки в демонстрации

Fig. 7. Normal operating mode of the plant in the demonstration

\section{Заключение}

В результате выполненной работы был предложен комплексный метод борьбы с гидратообразованием, который включает в себя два этапа: автоматизированный алгоритм анализа зон возможного гидратообразования и прогноз, а также предложенная технология борьбы с гидратами тепловым газодинамическим методом. На базе Томского политехнического университета в специально оборудованной лаборатории, где находится экспериментальный стенд, было проведено более пятисот практических экспериментов, направленных на проведение анализа эффективности работы установки. Проведен анализ степени отделения частиц льда от начальной его концентрации и скорости потока, оценка требуемой мощности теплоносителя, анализ времени восстановления температуры теплоносителя. Также было автоматизировано управление лабораторным стендом, подготовлено программное обеспечение для управления установкой при эксплуатации в промышленности. Так как в настоящее время на месторождениях газоконденсата качество добываемого флюида снижается за счет увеличения объемной составляющей воды, то вероятность образования гидратов в системе трубопроводов резко увеличивается. Следовательно, для нефтегазовой промышленности рассмотренная в статье установка является очень актуальной для применения и внедрения в технологические процессы. Для экспериментальной установки получен акт внедрения в Томский политехнический университет, в дальнейшем, после проведения дополнительных исследований и получения финансирования, планируется создание опытно-промышленного образца для нефтегазодобывающих компаний. 


\section{СПИСОК ЛИТЕРАТУРЫ}

1. Запорожец Е.П., Шостак Н.А. Гидраты. - Краснодар: ИД «Юг», 2017. - 460 с.

2. Ширшова А.В., Данько М.Ю. Рост и диссоциация газогидратов в водонефтяных эмульсиях // Известия вузов. Сер. Нефть и газ. - 2017. - № 5. - С. 95-101.

3. Abgrall R. Discrete equations for physical and numerical compressible multiphase mixtures // Journal of Computational Physics. - 2016. - V. 186. - P. 361-396.

4. Комисссаров Ю.А., Гордеев Л.С., Вент Д.П. Процессы и аппараты химической технологии. - М.: Химия, 2018. - 250 с.

5. Saurel R. Simple and efficient relaxation methods for interfaces separating compressible fluids, cavitating flows and shocks in multiphase mixtures // Journal of Computational Physics. 2017. - V. 228. - № 5. - P. 1678-1712.

6. Галимзянов М.Н. Математическое моделирование образования газовых гидратов // Вестник Башкирского университета. 2019. - T. 15. - № 3. - C. 570-574.

7. Carroll J. Natural gas hydrates. 4th ed. - Houston: Gulf Professional Publ., 2020. - 392 p.

8. Попов Д.Н., Варфоломеева О.И., Хворенков Д.А. Особенности неизотермических течений жидкости с переменными реологическими свойствами в каналах с местными сопротивлениями // Вестник Ижевского государственного технического университета. - 2016. - № 1. - С. 146-149.

9. Application of numerical signal differentiation methods to determine stationarity of a process / A.V. Maystrenko, A.A. Svetlakov, T.V. Gandzha, V.V. Dmitriev, N.V. Aksenova // Petroleum and Coal. - 2017. - V. 59. - № 3. - P. 311-318.

10. Ахметов Ю.М., Калимуллин Р.Р. Исследование гидродинамических и термодинамических процессов высоконапорного многофазного вихревого течения жидкости // Вестник Уфимского государственного авиационного технического университета. - 2016. - Т. 16. - № 2. - С. 163-168.

11. Запорожец Е.П., Зиберт Г.К. Регулярные процессы и оборудование в технологиях сбора, подготовки и переработки нефтяных и природных газов. - Краснодар: ИД «Юг», 2017. - 620 с.

12. Прахова М.Ю., Краснов А.Н. Методы и средства предотвращения гидратообразования на объектах газодобычи // Нефтегазовое дело. - 2016. - № 1. - С. 101-118.

13. Saurel R. Mathematical and numerical modeling of two phase compressible flows with micro inertia // Journal of Computational Physics. - 2016. - V. 175. - № 1. - P. 326-360.

14. Saeed A., Ruggeri B. Control of hydrates in natural gas during transportation. - Saarbrucken: Lambert Academic Publ., 2019. $96 \mathrm{p}$.

15. Волков П.В. Экспериментальное исследование аэромеханического метода для предотвращения гидратообразования при разработке газовых месторождений и установок комплексной подготовки газа // Нефть и газ 2019: Сборник трудов 73-й международной молодежной научной конференции. - 2019. T. 1. - C. $78-83$

16. Волков П.В. Экспериментальное исследование теплового газодинамического метода для предотвращения гидратообразования в газопроводах с использованием циклонов // Известия Томского политехнического университета. Инжиниринг георесурсов. - 2020. - Т. 331. - № 11. - C. 20-29.

17. Naseer M. Hydrate formation in natural gas pipelines // WIT Transactions on Engineering Sciences. - 2017. - V. 70. - P. 261-270.

18. Программно-аналитический комплекс для анализа зон возможного гидратообразования на участке трубопровода: свидетельство о государственной регистрации программы для ЭВМ №2020617523 Российская Федерация. № 202061664; заявл. 25.06.20; опубл. 08.07.20, Бюл. № 11. - 1 с.

19. Derevich I.V. The hydrodynamics and heat transfer and mass transfer of particles under conditions of turbulent flow of gas suspension in a pipe and in an axisymmetric jet // Journal of High Temperature. - 2016. - V. 40. - P. 78-91.

20. Fortin $\mathrm{G}$. A new roughness computation method and geometric accretion model for airfoil icing // Journal of Aircraft. - 2016. V. 41. - P. 119-127.

21. Букреев В.Г., Шандарова В.Г., Рулевский В.М. Нелинейная модель электроснабжения подводных объектов на основе изменения длины троса // Известия Томского политехнического университета. Инжиниринг георесурсов. - 2018. - Т. 329. № 11. - C. 114-123.

22. Программный комплекс для автоматизации работы модульной вставки борьбы с гидратообразованием тепловым газодинамическим методом: свидетельство о государственной регистрации программы для ЭВМ №2020660136 Российская Федерация. № 2020619336; заявл. 18.08.20; опубл. 28.08.20, Бюл. № 10. - 1 c.

23. Букреев В.Г., Шандарова В.Г., Рулевский В.М. Модель системы электропитания оборудования удаленной обработки // Известия Томского политехнического университета. Инжиниринг георесурсов. - 2018. - Т. 329. - № 4. - С. 119-131.

Поступила 10.03.2021 2.

\section{Информация об авторах}

Волков П.В., младший научный сотрудник АО ТомскНИПИнефть; младший научный сотрудник, Национальный исследовательский Томский политехнический университет.

Большунов A.B., аспирант отделения добычи нефти Инженерной школы природных ресурсов Национального исследовательского Томского политехнического университета.

Мостокалов К.А., аспирант отделения добычи нефти Инженерной школы природных ресурсов Национального исследовательского Томского политехнического университета. 
UDC 622.279 .8

\title{
METHODOLOGICAL AND ALGORITHMIC SUPPORT OF AN AUTOMATED EXPERIMENTAL INSTALLATION FOR REMOVING HYDRATES IN A GAS-CONDENSATE FLUID IN A PIPELINE
}

Pavel V. Volkov ${ }^{1,2}$ VolkovPV@tomsknipi.ru

\author{
Artem V. Bolshunov², \\ tpuiprgrnm@mail.ru \\ Kiril A. Mostokalov², \\ $555 @$ t-sk.ru \\ 1 TomskNIPIneft, \\ 72 Mira avenue, Tomsk, 634027, Russia. \\ 2 National Research Tomsk Polytechnic University, \\ 30, Lenin avenue, Tomsk, 634050, Russia.
}

The relevance of the study is caused by the fact that at present the problem of hydrate formation is quite acute in the global oil and gas industry. The accumulation of hydrates in the pipeline section or in certain units of hydrocarbon treatment devices can lead to disruption of the technological process, equipment failure, and decrease in the quality of finished products for the consumer. The main task is to timely determine the presence of hydrates in the pipeline cavity or correctly predict its formation and accumulation. There are many methods of combating hydrate formation, but they can be high-cost, ineffective or have a high hazard class. The article discusses a new way to eliminate hydrate formation and suggests a methodology for automating the process. The hardware part is considered as a simulation experimental stand for conducting experiments, checking analytical calculations and automation. The software part presents a technique for automating the determination of zones of possible hydrate formation, which allows better assessment, and a program for the automated operation of a pilot industrial sample in real conditions. Operation algorithms, emergency and normal shutdown algorithms are described. For a more accurate and quick analysis of problem areas of hydrate formation, as well as trouble-free operation of the unit, a rigorous algorithm was developed. The command system of the algorithm includes all emergency situations, possible errors, and limiting operating modes. The operation of all algorithms was tested in a practical way by simulating the system real operation and supplying conditional signals to the input of the installation software module.

The main aim of the research is to apply and analyze the operation of algorithmic support for the operation of an experimental plant for removal of hydrates formation from a gas-condensate fluid flow.

Object: autonomous modular installation for removing hydrate formation.

Methods: theoretical methods for substantiating the regularity of the flow of heat and mass transfer, calculation and analytical method for determining the quality of the developed software, practical methods for conducting experiments.

Results. The authors have built the laboratory stand on the basis of the National Research Tomsk Polytechnic University, carried out experiments for various gas flows with different thermobaric conditions, developed algorithmic support to automate the installation, tested the system.

Key words:

Hydrate formation, algorithm, software package, accumulation of hydrates, cyclone.

\section{REFERENCES}

1. Zaporozhets E.P. Gidraty [Hydrates]. Krasnodar, Yug Publ. house, 2017. $460 \mathrm{p}$.

2. Shirshova A.V. Growth and dissociation of gas hydrates in oilwater emulsions. Oil and gas, 2017, no. 5, pp. 95-101. In Rus.

3. Abgrall R., Saurel R. Discrete equations for physical and numerical compressible multiphase mixtures. Journal of Computational Physics, 2016, vol. 186, pp. 361-396.

4. Komissssarov Yu.A., Gordeev L.S., Vent D. P. Protsessy i apparaty khimicheskoy tekhnologii [Processes and devices of chemical technology]. Moscow, Khimiya Publ., 2018. 250 p.

5. Saurel R., Petitpas F., Berry R.A. Simple and efficient relaxation methods for interfaces separating compressible fluids, cavitating flows and shocks in multiphase mixtures. Journal of Computational Physics, 2017, vol. 228, no. 5, pp. 1678-1712.

6. Galimzyanov M.N. Mathematical modeling of the formation of gas hydrates. Herald of Bashkir University, 2018, vol. 15, no. 3 , pp. 570-574. In Rus.

7. Carroll J. Natural gas hydrates. $4^{\text {th }}$ ed. Houston, Gulf Professional Publ., 2020. 392 p.

8. Popov D.N., Varfolomeeva O.I., Khvorenkov D.A. Features of nonisothermal fluid flows with variable rheological properties in channels with local resistances. Herald of the Izhevsk State Technical University, 2016, no. 1, pp. 146-149. In Rus.

9. Maystrenko A.V., Svetlakov A.A., Gandzha T.V., Dmitriev V.V., Aksenova N.V. Application of numerical signal differentiation methods to determine stationarity of a process. Petroleum and Coal, 2017, vol. 59, no. 3, pp. 311-318.

10. Akhmetov Yu.M., Kalimullin R.R., Khakimov R.F., Konstantinov S.Yu., Tselishchev D.V. Investigation of the hydrodynamic and thermodynamic processes of high-pressure multiphase vortex fluid flow. Herald of Ufa State Aviation Technical University, 2016, vol. 16, no. 2, pp. 163-168. In Rus.

11. Zaporozhets E.P. Regularnye protsessy i oborudovanie $v$ technologiakh sbora, podgotovki i pererabotki neftyanykh i prirodnykh gazov [Regular processes and equipment in the technology of collection, preparation and processing of oil and natural gases]. Krasnodar, Yug Publ. house, 2017. $620 \mathrm{p}$.

12. Prakhova M.Yu., Krasnov A.N., Khoroshavina E.A., Shalovnikov E.A. Methods and means of preventing hydrate formation at gas production facilities. Oil and Gas Business, 2016, no. 1, pp. 101-118. In Rus.

13. Gavrilyuk S.L., Saurel R. Mathematical and numerical modeling of two phase compressible flows with micro inertia. Journal of Computational Physics, 2016, vol. 175, no. 1, pp. 326-360 
14. Saeed A., Ruggeri B. Control of hydrates in natural gas during transportation. Saarbrucken, Lambert Academic Publ., 2019. 96 p.

15. Volkov P.V. An experimental study of the aeromechanical method to prevent hydrate formation during the development of gas fields and integrated gas treatment plants. Oil and Gas, 2019, vol. 1, pp. 78-83. In Rus.

16. Volkov P.V., Bolshunov A.V. Experimental study of the thermal gas-dynamic method to prevent hydrate formation in gas pipelines using cyclones. Bulletin of the Tomsk Polytechnic University. Geo Assets Engineering, 2020, vol. 331, no. 11, pp. 20-29. In Rus.

17. Naseer M., Brandstatter W. Hydrate formation in natural gas pipelines. WIT Transactions on Engineering Sciences, 2017, vol. 70, pp. 261-270.

18. Volkov P.V. Programmno-analiticheskiy complex dlya analiza zon vozmozhnogo gidratoobrazovania na uchastke truboprovoda [Software-analytical complex for the analysis of zones of possible hydrate formation in the pipeline section]. Certificate of state registration of the computer program, no. 2020617523, 2020.

19. Derevich I.V. The hydrodynamics and heat transfer and mass transfer of particles under conditions of turbulent flow of gas sus- pension in a pipe and in an axisymmetric jet. Journal of High Temperature, 2016, vol. 40, no. 1, pp. 78-91.

20. Fortin G. A new roughness computation method and geometric accretion model for airfoil icing. Journal of Aircraft, 2016, vol. 41, no. 1, pp. 119-127.

21. Bukreev V.G., Shandarova E.B., Rulevskiy V.M. Nonlinear power supply model of submersible objects based on change in the cablerope length. Bulletin of the Tomsk Polytechnic University. Geo Assets Engineering, 2018, vol. 329, no. 11, pp. 114-123.

22. Volkov P.V. Programmny complex dlya avtomatizatsii raboti modulnoy vstavki borby s gidratoobrazovaniem teplovym gazodinamicheskim metodom [A software package for automating the work of a modular insert for combating hydrate formation by the thermal gas-dynamic method]. Certificate of state registration of a computer program, no. 2020660136, 2020.

23. Bukreev V.G., Shandarova E.B., Rulevskiy V.M. Power supply system model of remote processing equipment. Bulletin of the Tomsk Polytechnic University. Geo Assets Engineering, 2018, vol. 329 , no. 4, pp. 119-131.

Received: 10 March 2021.

\section{Information about the authors}

Pavel V. Volkov, junior researcher, TomskNIPIneft; junior researcher, National Research Tomsk Polytechnic University. Artem V. Bolshunov, postgraduate student, National Research Tomsk Polytechnic University.

Kiril A. Mostokalov, postgraduate student, National Research Tomsk Polytechnic University. 